\title{
Differential diagnosis and management of focal ground-glass opacities
}

\author{
M. Infante*, R.F. Lutman\#, S. Imparato\#, M. Di Rocco ", G.L. Ceresoli ${ }^{+}$, V. Torri', \\ E. Morenghi', F. Minuti ${ }^{+}$, S. Cavuto*, E. Bottoni*, F. Inzirillo*, U. Cariboni*, V. Errico*, \\ M.A. Incarbone*, G. Ferraroli*, G. Brambilla\#, M. Alloisio* and G. Ravasi*
}

ABSTRACT: Focal pulmonary ground-glass opacities (GGOs) can be associated with bronchioloalveolar carcinoma. The present retrospective study aimed to test the validity of a multistep approach to discriminate malignant from benign localised (focal) GGOs, identifies useful diagnostic features on computed tomography (CT), and suggests appropriate management guidelines.

A stepwise approach, including oral antibiotics, follow-up high-resolution CT (HRCT) 4060 days later and CT-guided core biopsy, was used. All cases with localised GGOs detected since 2001 were reviewed. CT features were described according to a structured scheme. In total, 40 patients were evaluated. Of these, 11 patients were diagnosed with benign GGOs, 19 patients had lung cancer and 10 were undetermined.

Nonpolygonal shape, apparent radial growth and clear-cut margins were associated with a malignant histology. The specificity of CT findings was low. Diagnostic accuracy increased after oral antibiotics, follow-up HRCT and percutaneous core biopsy. Overall, 18 patients underwent surgery for lung cancer.

In conclusion, malignant ground-glass opacities have a fairly typical appearance, but some benign lesions closely mimic their malignant counterparts. The stepwise approach adopted in the present study increased the diagnostic specificity and reduced time to definitive diagnosis. Segmentectomy might be the ideal resection volume for such tumours.

KEYWORDS: Antibiotics, diagnostic procedures, high-resolution computed tomography, management, pulmonary nodules, transthoracic needle aspiration biopsy

round-glass opacity (GGO) is a radiological term indicating an area of hazy increased lung opacity through which vessels and bronchial structures may still be seen. It is less opaque than consolidation, in which such structures are obscured [1]. Most commonly, diffuse GGOs are associated with widespread inflammatory or infiltrative lung disorders [2].

Focal GGOs, also called nonsolid or part-solid nodules [3], are circumscribed areas of hazy lung opacity. Their association with early-stage bronchioloalveolar carcinoma (BAC) was first reported in the 1990s by Japanese and Korean investigators [4, 5], shortly after the advent of low-dose spiral computed tomography (CT) for lung cancer screening. Since then, a number of publications have addressed the clinical significance of focal GGOs and their relationship with atypical adenomatous hyperplasia (AAH), BAC and invasive adenocarcinoma [6-10].

A number of such cases have come to the attention of the current authors since 2001, due to their ongoing lung cancer screening trial [11], and they were thus confronted with several issues: how to distinguish benign, nonevolving GGOs from those associated with BAC or adenocarcinoma; how to reach a definitive diagnosis in a reasonably short time; how to manage poor-risk surgical patients; whom and how to treat.

The current authors have retrospectively analysed a series of patients with localised GGOs, and the findings are herewith discussed in an attempt to establish practical guidelines for the management of these patients.

\section{PATIENTS AND METHODS}

\section{Study design}

All cases with localised GGOs, either screening detected or incidentally found from June 2001 to November 2007 at the Istituto Clinico Humanitas Hospital (Milan, Italy), were retrospectively reviewed.

A stepwise management scheme was used on a regular basis with pulmonary localised GGOs of
AFFILIATIONS

*Depts of Thoracic Surgery,

\#Radiology,

"Pathology,

${ }^{+}$Oncology, and

${ }^{\S}$ Clinical Research

IRCCS Istituto Clinico Humanitas, Milan, Italy.

CORRESPONDENCE

M. Infante

IRCCS Istituto Clinico Humanitas

Via Manzoni 56

20089 Rozzano

Milan

Italy

Fax:maurizio.infante@humanitas.it,

maurizio_infante@yahoo.it

Received:

March 272008

Accepted after revision:

November 132008

STATEMENT OF INTEREST

None declared. 
undetermined origin, according to screening trial management guidelines [11]. It consisted of the following steps: 1) oral antibiotics (levofloxacin $500 \mathrm{mg}$ daily for 8 days); 2) repeat high-resolution CT (HRCT) of the lesion with contiguous 1mm slice thickness reconstructions 40-60 days later; 3) a tissue diagnosis, if no regression had occurred.

The GGOs were described by two radiologists (R.F. Lutman and S. Imparato) blinded to the final pathological or clinical diagnosis, according to a structured scheme derived from the work of Li et al. [12]. Pathology and surgical reports, follow-up data and outcomes were also reviewed.

Incidentally found GGOs were included if there was no known history of respiratory infection, chest trauma, pulmonary or systemic disease that could explain their presence.

Diffuse GGOs, mild heterogeneity of lung parenchyma, GGOs associated with massive consolidation and centrilobular GGOs associated with bronchiolitis were excluded.

\section{Methods}

The shape of a GGO was classified as rounded, oval, lobulated (when gross indentations were visible along its borders) or polygonal (when all margins were roughly linear). Its surface could be defined as smooth or nonsmooth (finely or grossly irregular), while the margins were defined as smooth or irregular, and clear-cut or ill-defined in comparison with the surrounding normal lung parenchyma. A lesion was defined as a pure GGO when no solid component was visible, or a mixed GGO when a minimal ( $<25 \%$ of the GGO area) or major $(>25 \%$ of GGO area) solid density was visible.

If the lesion spread along the pulmonary lobular or segmental structures, a lobular/segmental distribution pattern was indicated, while if the GGO apparently extended through adjacent lobules in a centrifugal fashion, a radial growth pattern was indicated.

Multislice CT scanners and workstations were used for the evaluation of GGOs. Determinations of each CT feature were established by consensus readings.

Based on the first spiral CT findings, the lesions were initially categorised as possibly benign, undetermined or possibly neoplastic. After reviewing the cases, the radiologists formulated a tentative consensus diagnosis (inflammatory GGO, undetermined or lung cancer) according to the results of antibiotic trial and short-term follow-up HRCT. Radiologicalpathological correlations were made afterwards.

Pathological diagnoses were based on the World Health Organization 2004 criteria [13]. In the absence of a biopsy, GGOs were classified as benign if they had remained stable for at least 3 yrs.

\section{Statistical analysis}

Continuous data are presented as mean \pm SD, whereas categorical data are presented as numbers and percentages. Relationships between each single radiological or clinical feature and the final diagnosis were analysed using unpaired t-tests or the Fisher's exact test. A significance level of $5 \%$ was adopted. AAH was grouped together with benign lesions.

\section{RESULTS}

The current series of study subjects comprised 40 patients, 35 males and 5 females. In 29 patients, the GGOs were detected by screening, while in 11 cases they were found incidentally during investigation for an unrelated medical condition. The mean \pm SD age of the whole group was $67 \pm 6$ yrs, and $39(97 \%)$ were current $(n=17)$ or former $(n=22)$ smokers. Four patients had an associated synchronous solid lung cancer, which was resected in three patients. One patient had had a stage I lung cancer resected several years earlier.

Respiratory function tests were available for 21 patients: they were normal in eight patients and abnormal in 13. Out of 19 patients with lung cancer, 12 (63\%) had documented chronic obstructive and/or restrictive pulmonary disease on spirometry testing. Patient work-up, diagnostic procedures and final diagnoses are outlined in figure 1.

In each of $30(75 \%)$ patients, one lesion was found, while in 10 cases there were two or more lesions, either ipsilateral (two cases) or bilateral. The mean \pm SD age of patients with lung cancer was $68.9 \pm 7 \mathrm{yrs}$, while in those with benign lesions it was $65.4 \pm 7$ yrs. The difference was nonsignificant $(p=0.15)$.

The shape of malignant GGOs tended to be rounded, oval or grossly lobulated, e.g. nonpolygonal $(\mathrm{p}=0.006)$. Apparent radial growth was associated with neoplastic GGOs $(p=0.010)$; however, in seven $(27 \%)$ out of 26 cases it was observed in a benign lesion. A central or eccentric solid density (mixed GGO) was associated with malignancy in $75 \%$ of the cases, but overall the association between a solid component and lung cancer was nonsignificant $(p=0.27)$. Clear-cut margins, best appreciable on HRCT scans, were instead significantly associated with a malignant histology $(p=0.003)$,

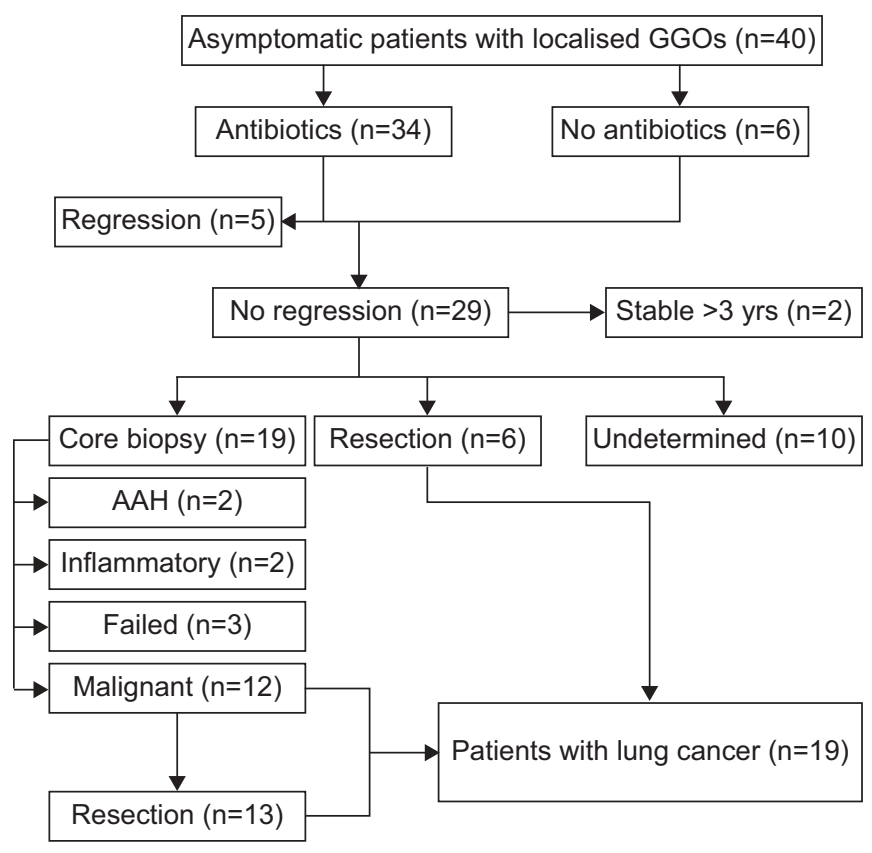

FIGURE 1. Diagram of patient management procedures. In six patients, the lesion was retrospectively visible in an earlier computed tomography scan, and antibiotics were not administered. GGOs: ground-glass opacities; $\mathrm{AAH}$ : atypical adenomatous hyperplasia. 

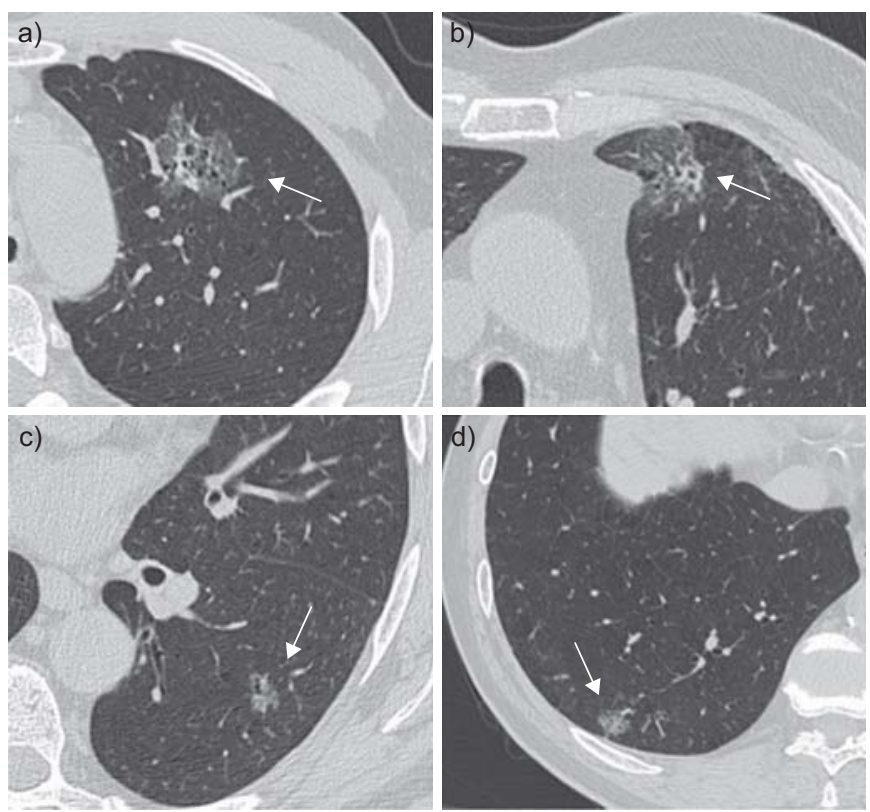

FIGURE 2. Benign and malignant ground-glass opacities (GGOs; arrows) with close resemblance. a) Persistent GGO in the left upper lobe, with irregular contour, clear-cut margins, a minimal solid component and tiny air spaces. Diagnosed as mixed-type adenocarcinoma. b) Similar lesion, with irregular contour, clear-cut margins, a minimal solid component and tiny air spaces, which persisted for $>3$ months after antibiotics. The core biopsy yielded inflammatory tissue. The lesion eventually regressed over several months (not shown). c) Persistent GGO in the right lower lobe, with irregular shape and minimal solid component. The core biopsy was suggestive of bronchioloalveolar carcinoma, which was confirmed by surgical resection. d) Similar lesion in the left lower lobe, with irregular shape and minimal solid component. The core biopsy yielded inflammatory tissue and fibrosis.

although they were also observed in benign GGOs (fig. 2). There was no demonstrable association with lung cancer risk for the number of lesions $(p=0.42)$, lesion diameter $(p=0.14)$ or surface characteristics $(p=0.26)$ in the current series. In table 1 , the sensitivity, specificity, accuracy and predictive values of selected CT features are given.

The diagnosis based only on the initial spiral CT was undetermined in $55 \%$ of the cases. A course of oral antibiotics ruled out five $(12.5 \%)$ cases with inflammatory lesions, as partial or complete regression was observed at repeat CT-scan after 2 months (fig. 3a). It is worth mentioning that six patients were evaluated on the basis of follow-up only (i.e. no antibiotics were given), as lesions were retrospectively visible in earlier CT scans.

The sensitivity, specificity, accuracy, positive and negative predictive values of the tentative diagnosis after antibiotics and/or follow-up HRCT were 1.00, 0.55, 0.86, 0.82 and 1.00, respectively, and the diagnosis remained undetermined in only $12 \%$ of the cases.

Percutaneous CT-guided core biopsy was carried out in 19 patients. It was indicative of a BAC or a mixed-type adenocarcinoma (i.e. adenocarcinoma with bronchioloalveolar features) in 12 patients, of an AAH in two (confirmed by resection in one and followed in the other), of an inflammatory lesion in two more, and was nondiagnostic in three (17\%) cases. Two of these underwent surgical resection based on clinical suspicion and were demonstrated to have multifocal BAC in one case and mixed-type adenocarcinoma in the other. The third declined further evaluation and was followed without signs of progression for 21 months. Complications were limited to mild pneumothorax in four patients (only one was drained) and mild haemoptysis in one.

Three patients refused percutaneous biopsy and further evaluation. One of them had a 20-mm mixed GGO in the left upper lobe at the time of detection by screening CT. He was admitted to the emergency department 4 yrs later in severe distress, with massive left lung atelectasis, and died shortly afterwards. A firm diagnosis could not be established. A second patient died of disseminated cancer of unknown origin 24 months later, and the third, who had a 9-mm pure rounded GGO in the right upper lobe, was still alive and well after 30 months.

Overall, the lesions of 10 patients were undetermined, either because the follow-up was $<3$ yrs, or core biopsy was refused or (in two cases) withheld due to significant comorbidities.

A positron emission tomography (PET) scan was obtained in 12 cases with histologically proven neoplastic GGOs prior to resection, and this was positive in three (25\%) cases.

\section{Surgery and outcomes}

Overall, $18(45 \%)$ patients underwent surgical resection for neoplastic GGOs. The mean $\pm \mathrm{SD}$ time from initial detection to

TABLE 1 Sensitivity, specificity, accuracy and predictive values of selected computed tomography features

\begin{tabular}{lccccccccc} 
Feature & TP & FP & TN & FN & Sensitivity & Specificity & Accuracy & PPV \\
\hline Multiple & 6 & 2 & 9 & 13 & $0.32(0.13-0.57)$ & $0.82(0.48-0.98)$ & $0.50(0.31-0.69)$ & $0.75(0.35-0.97)$ & $0.41(0.21-0.64)$ \\
Nonpolygonal & 19 & 6 & 5 & 0 & $1.00(0.83-1.00)$ & $0.45(0.16-0.76)$ & $0.80(0.61-0.92)$ & $0.76(0.55-0.91)$ & $1.00(0.48-1.00)$ \\
Mixed GGO & 13 & 4 & 7 & 6 & $0.68(0.43-0.87)$ & $0.64(0.31-0.89)$ & $0.67(0.47-0.83)$ & $0.76(0.50-0.93)$ & $0.54(0.25-0.81)$ \\
Nonsmooth & 17 & 6 & 5 & 2 & $0.89(0.67-0.99)$ & $0.45(0.17-0.77)$ & $0.73(0.54-0.88)$ & $0.74(0.52-0.90)$ & $0.71(0.29-0.96)$ \\
Radial growth & 19 & 7 & 4 & 0 & $1.00(0.82-1.00)$ & $0.36(0.11-0.69)$ & $0.77(0.58-0.90)$ & $0.73(0.52-0.88)$ & $1.00(0.40-1.00)$ \\
Clear-cut margin & 19 & 6 & 5 & 0 & $1.00(0.83-1.00)$ & $0.45(0.16-0.76)$ & $0.81(0.62-0.92)$ & $0.76(0.55-0.91)$ & $1.00(0.48-1.00)$ \\
Clear-cut and radial & 19 & 4 & 7 & 0 & $1.00(0.82-1.00)$ & $0.64(0.31-0.89)$ & $0.87(0.69-0.96)$ & $0.83(0.61-0.95)$ & $1.00(0.59-1.00)$ \\
\hline
\end{tabular}

Data are presented as $n$ or value (95\% confidence interval). TP: true positives; FP: false positives; TN: true negatives; FN: false negatives; PPV: positive predictive value NPV: negative predictive value; GGO: ground-glass opacity. 

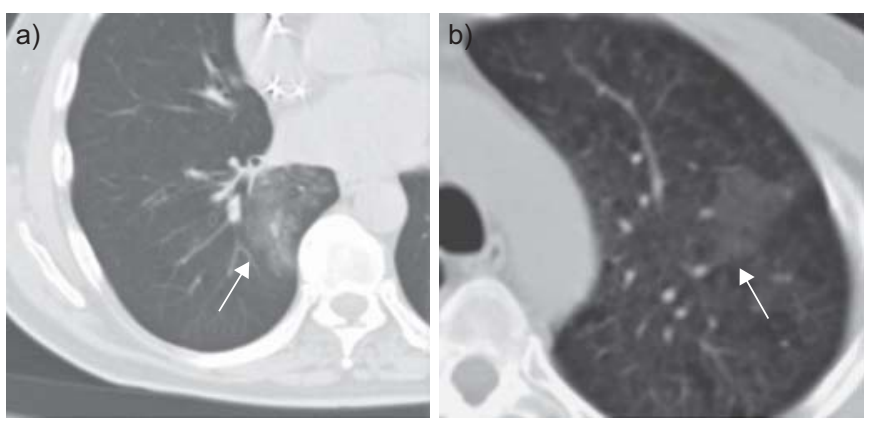

FIGURE 3. Benign ground-glass opacities (GGOs; arrows). a) Large GGO in the right lower lobe with irregular contour and subsegmental distribution. Following antibiotic treatment, the lesion regressed in 3 months. b) Persistent pure GGO in the left upper lobe with polygonal shape. This patient had a synchronous contralateral stage I solid adenocarcinoma, which was resected. The lesion remained stable for 46 months.

resection was $4.7 \pm 3.4$ months. One patient was operated on twice, $1 \mathrm{yr}$ apart, for two synchronous lesions (case No. 34). One patient underwent a middle lobectomy for a solid adenocarcinoma, together with bilateral wedge resections through a median sternotomy for multifocal mixed GGOs (mixed-type adenocarcinoma on histology).

No patient in the current series had a thoracotomy for an inflammatory lesion. However, one patient with a small mixed GGO in the left upper lobe underwent segmentectomy after a core biopsy had correctly diagnosed AAH pre-operatively. The management procedures in the present series are summarised in table 2.

Two patients developed recurrent lung cancer in the ipsilateral lung. The first did so 6 months following right lower lobectomy for stage I BAC. She eventually underwent reresection (right upper lobe segmentectomy), which demonstrated stage IIA mixed-type adenocarcinoma with one metastatic hilar lymphnode, and she was alive and well at the time of writing. The second recurrence was detected 30 months after left upper lobectomy for stage I mixed-type adenocarcinoma. Completion pneumonectomy was carried out and multifocal mixed-type adenocarcinoma was demonstrated in the resected specimen.

A third patient died with bone metastasis 10 months following right upper lobectomy for stage I mixed-type adenocarcinoma. He had undergone a left lower lobectomy 2 yrs earlier for a stage I solid adenocarcinoma.

\section{DISCUSSION}

A previously unknown radiological-pathological entity, localised BAC associated with focal GGO, is now encountered with relative frequency due to the widespread use of spiral $\mathrm{CT}$ for lung cancer screening and for routine pulmonary imaging studies. In the present retrospective study, a series of 40 cases with localised GGOs potentially associated with lung cancer was analysed, in the search for practical guidelines that could help reduce time to final diagnosis and treatment of lung cancer and at the same time avoid unnecessary anxiety in patients with benign GGOs. The current authors also tried to address some of the relevant management problems that may descend from finding such lesions, in particular regarding the indications and timing of surgery and the appropriate volume of surgical resection.

To summarise the current findings, the absence of a solid component, presence of a polygonal shape and ill-defined margins were associated with benign, nonevolving GGOs (fig. 3), while apparent radial growth, a nonpolygonal shape and clear-cut margins were associated with BAC or invasive adenocarcinoma. Malignancy was more likely with a mixed GGO pattern (positive predictive value 0.76), although the correlation did not reach statistical significance. The diameter of the lesions was, however, not associated with malignancy in the current series, possibly because some larger GGOs were inflammatory. The margins of the lesion were found to be especially important, and the current authors recommend that they should always be assessed in detail by HRCT.

The present findings are consistent with the observations of Li et al. [12], who reported that a rounded shape was more likely in malignant than in benign GGOs, and that a mixed GGO pattern was associated with malignancy in $85 \%$ of the cases. NAMBU et al. [14] similarly reported that $90 \%$ of malignant

TABLE 2 Patient management and pathological findings

\begin{tabular}{|c|c|c|c|c|c|c|}
\hline & \multicolumn{5}{|c|}{ Management } & \multirow[t]{2}{*}{ Total patients } \\
\hline & Lobectomy & Segmentectomy & Lob+We & Chemo/RT & FU & \\
\hline \multicolumn{7}{|l|}{ Pathology } \\
\hline Adeno/BAC & 6 & 3 & 2 & 1 & 0 & 12 \\
\hline $\mathrm{BAC}$ & 6 & 0 & 0 & 1 & 0 & 7 \\
\hline Undetermined & 0 & 0 & 0 & 0 & 10 & 10 \\
\hline Total procedures & 12 & 4 & 2 & 2 & 20 & 40 \\
\hline
\end{tabular}

One patient had two synchronous ground-glass opacities and was operated on twice. Lob: lobectomy; We: wedge resections; Chemo: chemotherapy; RT: radiotherapy; FU: follow-up; Adeno: adenocarcinoma; BAC: bronchioloalveolar carcinoma; AAH: atypical adenomatous hyperplasia. 
GGOs in their series had well-defined margins, but this feature was present in $50 \%$ of benign GGOs as well.

The current findings indicate that, with growing experience, it may become relatively easy to identify suspicious lesions based on the recognition of certain patterns, but it is not possible to reliably discriminate malignant from benign GGOs based on CT findings alone, as some benign lesions closely resemble their malignant counterparts (fig. 2).

Response to antibiotic trial is a simple criterion to rapidly exclude patients from further work-up, and the current authors therefore recommend it as the first management step. In GGOs persisting beyond 2 months after antibiotics, core biopsy under CT guidance yielded a meaningful tissue fragment and helped in the selection of patients for resection or follow-up in over $80 \%$ of the cases in the current series, and it is now commonly employed in the evaluation of such lesions. Core biopsy for GGOs has been reported to have a low complication rate, a positive predictive value of $97 \%$ and a negative predictive value of $75 \%$ [15]. Oral antibiotics, repeat HRCT and percutaneous core biopsy when indicated will delay appropriate surgery only by about 3 months. In the current authors' experience, the sensitivity of PET was low ( $25 \%$ overall), and similarly poor results have been reported by several other studies $[6,10]$. Therefore, the routine use of PET in the evaluation of suspicious GGOs is not recommended.

Among the present study patients, 65\% of focal GGOs for which a definitive diagnosis was made turned out to be associated with BAC or mixed-type adenocarcinoma after careful work-up, a finding consistent with the series of HENSCHKE et al. [3] in the USA, in which the malignancy rate was $18 \%$ for nonsolid nodules (i.e. pure GGOs) and $63 \%$ for part-solid nodules (i.e. mixed GGOs). KIM et al. [10] found the malignancy rate for persistent GGOs to be $75 \%$, whereas other Japanese or Korean groups observed malignancy rates in the range of $19-38 \%$, perhaps due to different study populations $[14,16,17]$.

Knowing that a relevant proportion of focal GGOs are associated with BAC or invasive adenocarcinoma, it would be logical to routinely proceed to biopsy and/or resection when one is encountered. However, at present it is impossible to tell which persistent GGOs will progress to aggressive disease with certainty, and how long it would take for them to do so. According to the study by TAKASHIMA et al. [9], about $25 \%$ of the lesions remain radiologically stable for several hundred days, even in the case of histologically proven BAC or adenocarcinoma. In another study, by KODAMA et al. [18], on 19 patients with pure GGOs, $42 \%$ of the lesions were radiologically unchanged after $26-48$ months. Lesions progressing so slowly may remain clinically silent for the whole life of the patient, representing possible examples of overdiagnosis. With respect to $\mathrm{AAH}$, which normally presents as a pure GGO of limited size, no data are available about the actual risk and timing of progression to more aggressive forms if left untreated. Therefore, especially with poor-risk surgical candidates and with small pure GGOs, some lesions might be carefully followed after thorough discussion with the patient, until signs of progression become evident.
When resection is chosen, treatment planning should take into account that a good correlation has been established between HRCT findings and invasiveness in malignant GGOs. BAC without stromal invasion predominates in pure GGOs, while invasive adenocarcinoma becomes more frequent in mixed GGOs as the solid component increases [9]. NAKATA et al. [19] observed that $87 \%$ of GGOs with no or a minimal solid component represented pure BAC, whereas 56-91\% of GGOs with a more abundant solid component represented invasive adenocarcinoma. Similarly, in a number of reports by Japanese authors, the likelihood of nodal metastasis was zero for tumours with no or minimal solid component on HRCT, and increased to $27-31 \%$ for those with a more evident solid component [19-24]. Larger GGOs with a solid component are more likely to be PET-positive, and a high standardised uptake value has been reported to correlate with stromal invasion and lymphatic spread [25]. A visible solid component in the preoperative HRCT scan should, therefore, warrant a more extensive resection, whereas a pure GGO could be approached more conservatively.

Treatment planning should also take into account that multifocality is a relatively common occurrence, with up to $25 \%$ of the patients harbouring multiple GGOs $[9,14]$. In the current series, six out of 19 patients with pathologically proven malignant GGOs had two or more lesions at the time of detection, and two more patients with a single GGO developed recurrent foci of carcinoma in the ipsilateral lung and were operated on again. Multiple resections may, therefore, be required immediately or over several years, thus calling for lung-sparing procedures. In fact, the prognosis after surgical resection for BAC associated with pure focal GGOs is excellent even after simple video-assisted thoracic surgery wedge resection, which would be inadequate for solid lung carcinomas [26-29]. The reason may be that pure BACs are true early lesions [13].

In a recent study, the cure rate of small early-stage tumours with anatomic segmentectomy was shown to be equivalent to that obtained with standard lobectomy, but with significantly less impact on post-operative respiratory function. Although the study was not randomised, it reported on over 500 patients in a controlled fashion [30]. Segmentectomy could, therefore, be an oncologically acceptable operation for small early-stage lung cancer, allowing for adequate resection margins, removal of the local lymphatics and preservation of functional lung tissue at the same time. The current authors therefore advocate it for GGOs, especially for those showing a minor solid component. If segmentectomy is difficult to perform with adequate margins or if the solid component is greater than $50 \%$, a standard lobectomy would be the preferred treatment. Simultaneous wedge resections are acceptable for multiple synchronous GGOs.

Pre-operative percutaneous hook-wire marking is always carried out in the current authors' centre (Istituto Clinico Humanitas Hospital) when a limited resection is planned, as focal GGOs may not be palpable at all [16].

The current study is a retrospective, single-institution study on a relatively small series of patients. Despite these limitations, the present study reproduces the findings reported previously 
and supports a simple multistep approach to these patients, which can be applied regardless of personal experience with this entity.

In conclusion, while ground-glass opacities with selected features should be considered clearly suspicious, no single radiological trait is $100 \%$ accurate and predictive, and some benign lesions closely mimic their malignant counterparts. A stepwise management protocol, based on oral antibiotics, short-term follow-up with high-resolution computed tomography and percutaneous core biopsy, increases the diagnostic accuracy over low-dose computed tomography alone and reduces time to definitive diagnosis without delaying appropriate treatment significantly. Segmentectomy might be the ideal resection volume for such tumours when feasible. The management of individual patients should be personalised.

\section{ACKNOWLEDGEMENTS}

The authors wish to thank I. Filomeno (Dept of Thoracic Surgery, IRCCS Istituto Clinico Humanitas, Milan, Italy) for her assistance in coordinating patient recruitment and followup procedures and R. Roberts (Dept of Clinical Research, IRCCS Istituto Clinico Humanitas) for kindly reviewing the English text.

\section{REFERENCES}

1 Hansell DM, Bankier AA, MacMahon H, McLoud TC, Müller NL, Remy J. Fleishner Society: glossary of terms for thoracic imaging. Radiology 2008; 246: 697-722.

2 Collins J, Stern EJ. Ground-glass opacity at CT: the ABCs. AJR Am J Roentgenol 1997; 169: 355-367.

3 Henschke CI, Yankelevitz DF, Mirtcheva R, et al. CT screening for lung cancer: frequency and significance of part-solid and nonsolid nodules. AJR Am J Roentgenol 2002; 178: 1053-1057.

4 Kushihashi T, Munechika H, Ri K, et al. Bronchioloalveolar adenoma of the lung: CT-pathologic correlation. Radiology 1994; 193: 789-793.

5 Jang HJ, Lee KS, Kwon OJ, Rhee CH, Shim YM, Han J. Bronchioloalveolar carcinoma: focal area of ground-glass attenuation at thin-section CT as an early sign. Radiology 1996; 199: 485-488.

6 Gandara DR, Aberle D, Lau D, et al. Radiographic imaging of bronchioloalveolar carcinoma: screening, patterns of presentation and response assessment. J Thorac Oncol 2006; 1: Suppl. 9, S20-S26.

7 Nakata M, Saeki H, Takata I, et al. Focal ground-glass opacity detected by low-dose helical CT. Chest 2002; 121 : 1464-1467.

8 Kakinuma R, Ohmatsu H, Kaneko M, et al. Progression of focal pure ground-glass opacity detected by low-dose helical computed tomography screening for lung cancer. $J$ Comput Assist Tomogr 2004; 28: 17-23.

9 Takashima S, Maruyama Y, Hasegawa M, et al. CT findings and progression of small peripheral lung neoplasms having a replacement growth pattern. AJR Am J Roentgenol 2003; 180: 817-826.

10 Kim HY, Shim YM, Lee KS, Han J, Yi CA, Kim YK. Persistent pulmonary nodular ground-glass opacity at thin-section CT: histopathologic comparisons. Radiology 2007; 245: 267-275.

11 Infante $\mathrm{M}$, Lutman FR, Cavuto $\mathrm{S}$, et al. Lung cancer screening with spiral CT: baseline results of the randomized DANTE trial. Lung Cancer 2008; 59: 355-363.

12 Li F, Sone S, Abe H, MacMahon H, Doi K. Malignant versus benign nodules at CT screening for lung cancer: comparison of thin-section CT findings. Radiology 2004; 233: 793-798.

13 Travis WD, Garg K, Franklin WA, et al. Evolving concepts in the pathology and computed tomography imaging of lung adenocarcinoma and bronchioloalveolar carcinoma. $J$ Clin Oncol 2005; 23: 3279-3287.

14 Nambu A, Araki T, Taguchi Y, et al. Focal area of groundglass opacity and ground-glass opacity predominance on thin-section CT: discrimination between neoplastic and non-neoplastic lesions. Clin Radiol 2005; 60: 1006-1017.

15 Kim TJ, Lee JH, Lee CT, et al. Diagnostic accuracy of CTguided core biopsy of ground-glass opacity pulmonary lesions. AJR Am J Roentgenol 2008; 190: 234-239.

16 Oh JY, Kwon SY, Yoon HI, et al. Clinical significance of a solitary ground-glass opacity (GGO) lesion of the lung detected by chest CT. Lung Cancer 2007; 55: 67-73.

17 Ohtsuka T, Watanabe K, Kaji M, Naruke T, Suemasu K. A clinicopathological study of resected pulmonary nodules with focal pure ground-glass opacity. Eur J Cardiothorac Surg 2006; 30: 160-163.

18 Kodama K, Higashiyama M, Yokouchi H, et al. Natural history of pure ground-glass opacity after long-term follow-up of more than 2 years. Ann Thorac Surg 2002; 73: 386-392.

19 Nakata M, Sawada S, Yamashita M, et al. Objective radiologic analysis of ground-glass opacity aimed at curative limited resection for small peripheral non-small cell lung cancer. J Thorac Cardiovasc Surg 2005; 129: 1226-1231.

20 Asamura H, Suzuki K, Watanabe S, Matsuno Y, Maeshima A, Tsuchiya R. A clinicopathological study of resected subcentimeter lung cancers: a favorable prognosis for ground glass opacity lesions. Ann Thorac Surg 2003; 76: 1016-1022.

21 Matsuguma H, Yokoi K, Anraku M, et al. Proportion of ground-glass opacity on high-resolution computed tomography in clinical T1 N0 M0 adenocarcinoma of the lung: a predictor of lymph node metastasis. J Thorac Cardiovasc Surg 2002; 124: 278-284.

22 Suzuki K, Kusumoto M, Watanabe S, Tsuchiya R, Asamura H. Radiologic classification of small adenocarcinoma of the lung: radiologic-pathologic correlation and its prognostic impact. Ann Thorac Surg 2006; 81: 413-419.

23 Okada M, Nishio W, Sakamoto $T$, et al. Correlation between computed tomographic findings, bronchioloalveolar carcinoma component, and biologic behavior of small-sized lung adenocarcinomas. J Thorac Cardiovasc Surg 2004; 127: 857-861.

24 Ohde Y, Nagai K, Yoshida J, et al. The proportion of consolidation to ground-glass opacity on high resolution $\mathrm{CT}$ is a good predictor for distinguishing the population of non-invasive peripheral adenocarcinoma. Lung Cancer 2003; 42: 303-310.

25 Okada M, Tauchi S, Iwanaga K, et al. Associations among bronchioloalveolar carcinoma components, positron emission tomographic and computed tomographic findings, 
and malignant behavior in small lung adenocarcinomas. J Thorac Cardiovasc Surg 2007; 133: 1448-1454.

26 Watanabe S, Watanabe T, Arai K, Kasai T, Haratake J, Urayama $\mathrm{H}$. Results of wedge resection for focal bronchioloalveolar carcinoma showing pure ground-glass attenuation on computed tomography. Ann Thorac Surg 2002; 73: 1071-1075.

27 Nakamura H, Saji H, Ogata A, Saijo T, Okada S, Kato H. Lung cancer patients showing pure ground-glass opacity on computed tomography are good candidates for wedge resection. Lung Cancer 2004; 44: 61-68.
28 Yamada S, Kohno T. Video-assisted thoracic surgery for pure ground-glass opacities $2 \mathrm{~cm}$ or less in diameter. Ann Thorac Surg 2004; 77: 1911-1915.

29 Mun M, Kohno T. Efficacy of thoracoscopic resection for multifocal bronchioloalveolar carcinoma showing pure ground-glass opacities of $20 \mathrm{~mm}$ or less in diameter. Thorac Cardiovasc Surg 2007; 134: 877-882.

30 Okada M, Koike T, Higashiyama M, Yamato Y, Kodama K, Tsubota N. Radical sublobar resection for small-sized nonsmall cell lung cancer: a multicenter study. J Thorac Cardiovasc Surg 2006; 132: 769-775. 